\title{
Morbidade materna grave em hospitais públicos de Ribeirão Preto, São Paulo, Brasil
}

\section{Severe maternal morbidity in public hospitals in Ribeirão Preto, São Paulo State, Brazil}

\author{
Morbilidad materna grave en hospitales públicos \\ de Ribeirão Preto, São Paulo, Brasil
}

\author{
Magna Santos Andrade 1 \\ Livia Pimenta Bonifácio 2 \\ Jazmin Andrea Cifuentes Sanchez 2 \\ Livia Oliveira-Ciabati 2 \\ Fabiani Spessoto Zaratini 2 \\ Ana Carolina Arruda Franzon 2 \\ Vicky Nogueira Pileggi 2 \\ Giordana Campos Braga 2 \\ Mariana Fernandes 2 \\ Carolina Sales Vieira 2 \\ João Paulo Souza 2 \\ Elisabeth Meloni Vieira 2
}

\section{Resumo}

Este estudo objetivou investigar a ocorrência de morbidade materna grave, os critérios diagnósticos mais frequentes e a qualidade da assistência obstétrica nos hospitais públicos de Ribeirão Preto, São Paulo, Brasil. Trata-se de uma pesquisa quantitativa de vigilância das morbidades maternas graves, utilizando os critérios da Organização Mundial da Saúde (OMS) para condições potencialmente ameaçadoras à vida e near miss materno. Os casos foram identificados entre 1o de agosto de 2015 e 2 de fevereiro de 2016. Participaram 259 mulheres com morbidade materna grave (condições potencialmente ameaçadoras à vida/near miss materno) durante o ciclo gravídico-puerperal, internadas para o parto nas quatro instituições públicas com assistência obstétrica no município. Realizou-se análise descritiva a partir das frequências absolutas e relativas dos critérios diagnósticos para condições potencialmente ameaçadoras à vida e near miss materno, além da descrição das mulheres pesquisadas (caracterização sociodemográfica, antecedentes obstétricos, assistência pré-natal e ao parto). Indicadores de qualidade da assistência estabelecidos pela OMS a partir da morbimortalidade também foram calculados. Ocorreram 3.497 partos, 3.502 nascidos vivos em todos os hospitais do município, 2 mortes maternas e 19 near miss materno. A razão do near miss materno foi de 5, 4 casos por 1.000 nascidos vivos, e a razão de mortalidade materna foi de 57,1 óbitos por 100 mil nascidos vivos. A taxa de mortalidade entre os casos com desfecho materno grave (somados os casos de near miss materno e morte materna) foi de 9,5\%. Observou-se importante frequência de casos de condições potencialmente ameaçadoras à vida e near miss materno no presente estudo. A ocorrência de óbitos por causas hemorrágicas mostra a necessidade de melhoria da qualidade da assistência prestada. Os resultados poderão contribuir para o aprimoramento da política local de assistência obstétrica.
Correspondência

M. S. Andrade

Universidade do Estado da Bahia.

Rod. Lomanto Jr., BR $407 \mathrm{Km} \mathrm{127,} \mathrm{s/n,} \mathrm{Senhor} \mathrm{do} \mathrm{Bonfim,} \mathrm{BA}$ 48970-000, Brasil.

magnaenf@yahoo.com.br

1 Universidade do Estado da Bahia, Senhor do Bonfim, Brasil.

2 Universidade de São Paulo, Ribeirão Preto, Brasil. 


\section{Introdução}

A morte de mulheres no período gravídico-puerperal é um grave problema de saúde pública que atinge principalmente as regiões de baixa e média rendas. Entre os anos de 1990 e 2015 houve uma diminuição dos óbitos maternos em todo o mundo, mas esta redução ocorreu de maneira distinta ao comparar as diversas regiões 1 . Entretanto, o decréscimo mundial dos indicadores de óbitos maternos ainda é lento, o que é comprovado pelo fato de apenas nove países terem alcançado a redução de 75\% das mortes maternas entre os anos de 1990 e 2015, proposta pelos Objetivos de Desenvolvimento do Milênio 2,3.

A distribuição dos casos de mortes maternas em números absolutos difere drasticamente entre as regiões de alta e baixa rendas, tendo como exemplo a Finlândia que em 2015 apresentou três óbitos maternos por 100 mil nascidos vivos, já Serra Leoa alcançou 1.360 óbitos por 100 mil nascidos vivos 4 .

Para a redução da mortalidade materna é relevante conhecer os aspectos relacionados a esses casos. Todavia, o estudo apenas dos óbitos não é o mais adequado, devido ao reduzido número absoluto do acontecimento nas regiões com adequado desenvolvimento social e econômico. Além disso, a produção de informações de má qualidade sobre os óbitos maternos (erros de preenchimento ou informações incompletas dos relatórios médicos, definição equivocada da causa básica da morte e uso incorreto da Classificação Internacional de Doenças) resulta em informações não confiáveis e subestimação dos indicadores de morte 1 .

Nessa perspectiva, a Organização Mundial da Saúde (OMS) recomenda a realização de estudos sobre os casos de mulheres com condições potencialmente ameaçadoras à vida ou aquelas que quase morreram, mas sobreviveram a uma complicação no período da gestação, parto e até 42 dias de pós -parto conhecido como near miss materno. As condições potencialmente ameaçadoras à vida são casos menos graves e representam uma condição que geralmente ocorre antes do near miss materno no que se refere à gravidade, e este, muitas vezes precede a morte materna 5 .

$\mathrm{O}$ maior número de complicações maternas graves, em comparação à mortalidade materna, permite conhecer a qualidade do cuidado obstétrico ofertado pelos serviços de saúde e determina a incidência das complicações maternas graves que podem levar à morte 6 .

Em 2009, a OMS estabeleceu um conjunto de critérios de gravidade para a identificação dos casos de near miss materno e condições potencialmente ameaçadoras à vida. O uso dessa classificação tem a vantagem de padronizar a metodologia para a identificação de casos graves, o que permite a comparação da assistência para gestantes e puérperas ao longo do tempo e em qualquer local, independentemente do nível de desenvolvimento da localidade pesquisada 5,7 .

Um estudo realizado em 27 hospitais brasileiros identificou uma razão de near miss materno de 9,37 casos por mil nascidos vivos 8 , resultado que se aproxima do valor encontrado em pesquisas realizadas em países do Oriente Médio, Ásia, África e América Latina com 8,3 casos de near miss materno por mil nascidos vivos 9 .

Os casos de near miss materno compartilham características que são semelhantes às mortes maternas, por isto, o estudo das mulheres que desenvolvem near miss no período gravídico-puerperal pode fornecer informações importantes sobre as dificuldades na assistência obstétrica no momento da complicação 10, além da vantagem de entrevistar diretamente as mulheres, o que possibilita a coleta de informações mais detalhadas acerca do adoecimento e cuidado recebido 11.

A existência de dados fidedignos sobre as morbidades maternas graves pode contribuir para a tomada de decisão em todos os níveis assistenciais. Essas informações possibilitam a adequada alocação de recursos, o planejamento e a implantação de intervenções para a redução dos indicadores de morbimortalidade 12 .

O presente estudo objetivou investigar a ocorrência de morbidade materna grave, os critérios diagnósticos mais frequentes e a qualidade da assistência obstétrica nos hospitais públicos de Ribeirão Preto, São Paulo, Brasil. 


\section{Métodos}

\section{Desenho do estudo}

Trata-se de uma pesquisa quantitativa de vigilância das morbidades maternas graves.

\section{População, local e período de estudo}

O presente trabalho foi um componente da pesquisa denominada Pré-natal no Celular (PRENACEL), um ensaio clínico aleatorizado por conglomerados, realizado com mulheres que fizeram pré-natal em 20 unidades básicas de saúde (UBS) de Ribeirão Preto. As UBS foram aleatorizadas em dois grupos: 10 unidades para receber a intervenção e 10 como controle 13 .

O PRENACEL consistiu no desenvolvimento de um sistema de distribuição de conteúdo por meio de mensagens curtas de texto via celular (SMS - short message service) e avaliou se a utilização deste tipo de serviço direcionado para as gestantes aumenta a adesão às práticas recomendadas de cuidado pré-natal 13.

Para a obtenção dos dados referentes ao impacto da intervenção e aspectos relacionados às morbidade materna grave (condições potencialmente ameaçadoras à vida e near miss materno), as mulheres que tiveram partos nas quatro maternidades públicas da cidade de Ribeirão Preto (Centro de Referência da Saúde da Mulher - Mater, Santa Casa de Misericórdia de Ribeirão Preto, Maternidade Cidinha Bonini - Universidade de Ribeirão Preto e Hospital das Clínicas da Faculdade de Medicina de Ribeirão Preto - Universidade de São Paulo) foram entrevistadas após o parto.

As maternidades pesquisadas totalizam todas as instituições que oferecem assistência ao parto pelo Sistema Único de Saúde (SUS) em Ribeirão Preto, município localizado no nordeste do Estado de São Paulo, considerada uma região rica, com Índice de Desenvolvimento Humano de 0,8 em 2010 e população de 682.302 habitantes no ano de 2017 (Instituto Brasileiro de Geografia e Estatística. População e Economia. https://cidades.ibge.gov.br/brasil/sp/ribeirao-preto/panorama, acessado em 10/Mar/2019).

A coleta de dados ocorreu entre 3 de agosto de 2015 e 2 de fevereiro de 2016.

Os critérios de inclusão no estudo de vigilância foram: internação em uma das quatro maternidades públicas do município; ter 18 anos ou mais; estar no período de pós-parto; desenvolver morbidade materna grave (condições potencialmente ameaçadas à vida ou near miss materno) na gestação, parto ou até 42 dias de pós-parto, segundo dados do prontuário e de acordo com os critérios estabelecidos pela OMS em 2009 5. Com isso, paralelamente à coleta de dados das mulheres que realizaram o prénatal em uma das 20 UBS integrantes do estudo PRENACEL, toda e qualquer mulher que desenvolveu condições potencialmente ameaçadas à vida ou near miss materno também foi entrevistada.

\section{Variáveis, fontes de dados e mensuração}

Durante seis meses, assistentes de pesquisa realizaram visitas diárias às quatro maternidades. Os prontuários das mulheres internadas no pós-parto foram revisados e os provedores de cuidados de saúde foram abordados na busca de mulheres elegíveis. Buscava-se nos prontuários a presença de qualquer um dos critérios estabelecidos pela OMS para condições potencialmente ameaçadoras à vida ou near miss materno, a fim de identificar os casos de morbidade materna grave 5.

Para evitar perdas desses casos, foi criado um formulário com todos os critérios de classificação. Paralelamente, na caderneta da gestante (documento da mulher com dados do pré-natal), era identificada a UBS onde fez o pré-natal, para verificar se a unidade fazia parte do estudo PRENACEL.

As variáveis utilizadas neste trabalho foram obtidas do instrumento de coleta de dados do PRENACEL, preenchido com base em dados do prontuário, caderneta da gestante e entrevista face a face 13. Todas as gestantes integrantes do estudo de vigilância, participantes ou não do estudo PRENACEL, foram entrevistadas nas maternidades no mesmo período da coleta de dados.

As variáveis utilizadas para a caracterização das mulheres foram: sociodemográficas (idade, escolaridade, situação marital, raça/cor, se exerce trabalho remunerado, chefe de família e classe social definida pelo Critério Brasil - Associação Brasileira de Empresas de Pesquisa. http://www.abep.org/ 
criterio-brasil, acessado em 10/Mar/2019); antecedentes obstétricos (gestações e partos anteriores, cesarianas e partos vaginais prévios, abortos prévios, natimortos anteriores, último intervalo interpartal e complicações em gestações anteriores); assistência pré-natal e parto (planejamento da gestação, realização do pré-natal, número de consultas, pré-natal adequado, idade gestacional na primeira consulta, local do pré-natal, gestante de risco, risco atual devido à hipertensão, diabetes, sangramento e infecção urinária, tipo de gestação, tipo de parto e presença de trabalho de parto).

A variável "pré-natal adequado" foi elaborada baseando-se nas recomendações do Ministério da Saúde. Considerou-se o mínimo de consultas estabelecidas para cada período gestacional (mínimo de 6 consultas, sendo a primeira realizada até o final do primeiro trimestre, 2 no segundo e 3 no terceiro trimestre) 14 . Todas as gestantes integrantes do estudo de vigilância, participantes ou não do estudo PRENACEL, foram entrevistadas nas maternidades, no mesmo período de coleta de dados.

O formulário do PRENACEL foi testado por meio de um estudo piloto conduzido antes de iniciar a coleta de dados (julho de 2015). A equipe de coleta foi formada por oito entrevistadoras que receberam treinamento prévio e divididas em quatro grupos para a atuação em cada unidade hospitalar. Em cada instituição, uma supervisora era responsável pelo campo de coleta, realizando a verificação dos instrumentos para avaliar possíveis erros de preenchimento. Em casos de erros de informação, o questionário retornava para a entrevistadora que voltava a campo para a correção das inconsistências.

\section{Desfechos}

Os desfechos primários do estudo foram a descrição da frequência dos casos de morbidade materna grave (condições potencialmente ameaçadoras à vida e near miss materno).

\section{Tamanho amostral}

Para a presente análise de vigilância das complicações maternas graves, todos os casos elegíveis de morbidade materna grave identificados durante o período da coleta de dados foram incluídos, independentemente de ser oriundo ou não das UBS pesquisadas pelo estudo PRENACEL.

\section{Análise estatística}

Os dados foram digitados no Research Electronic Data Capture (REDCap. https://www.project-red cap.org/). O banco de dados foi transportado para o software Stata versão 9.1 (https://www.stata. com), processado e analisado. Em seguida, buscaram-se as inconsistências no banco de dados usando um conjunto de regras de validação/verificação cruzada. Os erros encontrados foram corrigidos por meio da verificação do formulário de papel e checagem do prontuário hospitalar. Em casos específicos, em que as inconsistências não puderam ser solucionadas, as variáveis inconsistentes foram anuladas e consideradas como informação faltante.

Inicialmente, foi realizada análise descritiva com base nas frequências simples e absolutas dos critérios diagnósticos para a classificação de condições potencialmente ameaçadoras à vida e near miss materno, além da descrição das mulheres pesquisadas valendo-se das variáveis sociodemográficas, antecedentes obstétricos, assistência pré-natal e ao parto.

Os indicadores de qualidade da assistência estabelecidos pela OMS baseando-se na morbidade e mortalidade foram calculados: razão de near miss materno (número de near miss materno por mil nascidos vivos), razão de desfecho materno grave (número de near miss materno somado às mortes maternas por mil nascidos vivos), razão entre near miss materno e morte materna (razão entre o total de near miss materno e o total de óbitos maternos), taxa de mortalidade entre os casos com desfecho materno grave (total de mortes maternas pelo total de desfechos maternos graves em porcentagem), razão de mortalidade materna - RMM (número de mortes maternas por 100 mil nascidos vivos) 5 . Além disso, também calculou-se a frequência de mulheres com condições potencialmente ameaçadoras à vida por mil nascidos vivos. 


\section{Aspectos éticos}

Este trabalho foi aprovado pelo Comitê de Ética em Pesquisa do Centro de Saúde Escola da Faculdade de Medicina de Ribeirão Preto, Universidade de São Paulo com protocolo no 971.458. Todas as participantes do estudo assinaram o Termo de Consentimento Livre e Esclarecido.

\section{Resultados}

Durante os seis meses de coleta de dados ocorreram 3.497 partos e 3.502 nascidos vivos nas maternidades públicas pesquisadas. Dentre esse total, foram identificados 259 casos de morbidade materna grave, com 19 casos de near miss materno e 2 mortes maternas.

Foram calculados os indicadores de morbidade e mortalidade materna entre mulheres atendidas nos hospitais públicos de Ribeirão Preto. Para cada mil nascidos vivos ocorreram 5,4 casos de near miss materno, 6 desfechos maternos graves, a taxa de mortalidade entre os casos de desfecho materno grave foi de 9,5\% e 57,1 óbitos maternos por 100 mil nascidos vivos (Tabela 1).

Ocorreram duas mortes maternas, uma cuja causa básica foi o choque circulatório, e as causas antecedentes foram hemorragia intrauterina e complicações pós-cesárea. O outro óbito teve como causa da morte o choque hipovolêmico ocorrido no pós-parto imediato.

Os principais critérios diagnósticos entre os casos de condições potencialmente ameaçadoras à vida foram pré-eclâmpsia grave (21,7 por mil nascidos vivos) e hipertensão grave (18,4 por mil nascidos vivos). Entre os casos de near miss materno, os critérios mais frequentes foram choque e transfusão $\geq 5$ unidades de concentrado de hemácias (1,7 caso por mil nascidos vivos) (Tabela 2). Os diagnósticos foram mutuamente excludentes e $49 \%$ das mulheres com complicações apresentaram mais de um critério de morbidade.

As mulheres com morbidade materna grave possuíam as seguintes características sociodemográficas: menos de 35 anos de idade (79,6\%), 8 anos ou mais de escolaridade (79,4\%), pretas/pardas (64,5\%), realizavam trabalho remunerado $(50,2 \%)$ e pertenciam à classe média (classe C) $(59,8 \%)$ (Tabela 3 ). Em relação aos antecedentes obstétricos, as mulheres com morbidade materna grave tinham 1 ou 2 partos anteriores $(44,4 \%)$, entre os partos prévios predominaram os vaginais (58\%), a ausência de abortos $(72,4 \%)$ ou natimortos $(96,7 \%)$ prévios, último intervalo interpartal $\geq 2$ anos $(89,7 \%)$ e ausência de complicações em gestações anteriores $(62,4 \%)$ (Tabela 4). Sobre a assistência ao pré-natal e ao parto, a gestação atual não foi planejada $(57,4 \%)$, realizaram pré-natal $(88,4 \%)$, pré-natal com 6 ou mais consultas $(74,2 \%)$, início do pré-natal no 1o trimestre $(67,7 \%)$, gestação de risco $(65,6 \%)$ e cesárea atual $(59,6 \%)$ (Tabela 5).

\section{Tabela 1}

Indicadores de morbidade e mortalidade materna entre mulheres atendidas nos hospitais públicos do Município de Ribeirão Preto, São Paulo, Brasil, 2015/2016.

\begin{tabular}{|c|c|}
\hline Indicadores & Razões \\
\hline Razão de condição potencialmente ameaçadoras à vida (/1.000 nascidos vivos) & 68,5 \\
\hline Razão de near miss materno (/1.000 nascidos vivos) & 5,4 \\
\hline Razão de desfecho materno grave (near miss materno + mortalidade materna/1.000 nascidos vivos) & 6,0 \\
\hline Razão entre near miss materno e mortalidade materna (near miss materno:1 mortalidade materna) & 9,5 \\
\hline Índice de mortalidade (mortalidade materna/near miss materno + mortalidade materna em \%) & 9,5 \\
\hline Razão de morte materna (mortalidade materna/100.000 nascidos vivos) & 57,1 \\
\hline
\end{tabular}


Tabela 2

Distribuição dos critérios de condições potencialmente ameaçadoras à vida e near miss materno entre mulheres atendidas nos hospitais públicos do Município de Ribeirão Preto, São Paulo, Brasil, 2015/2016.

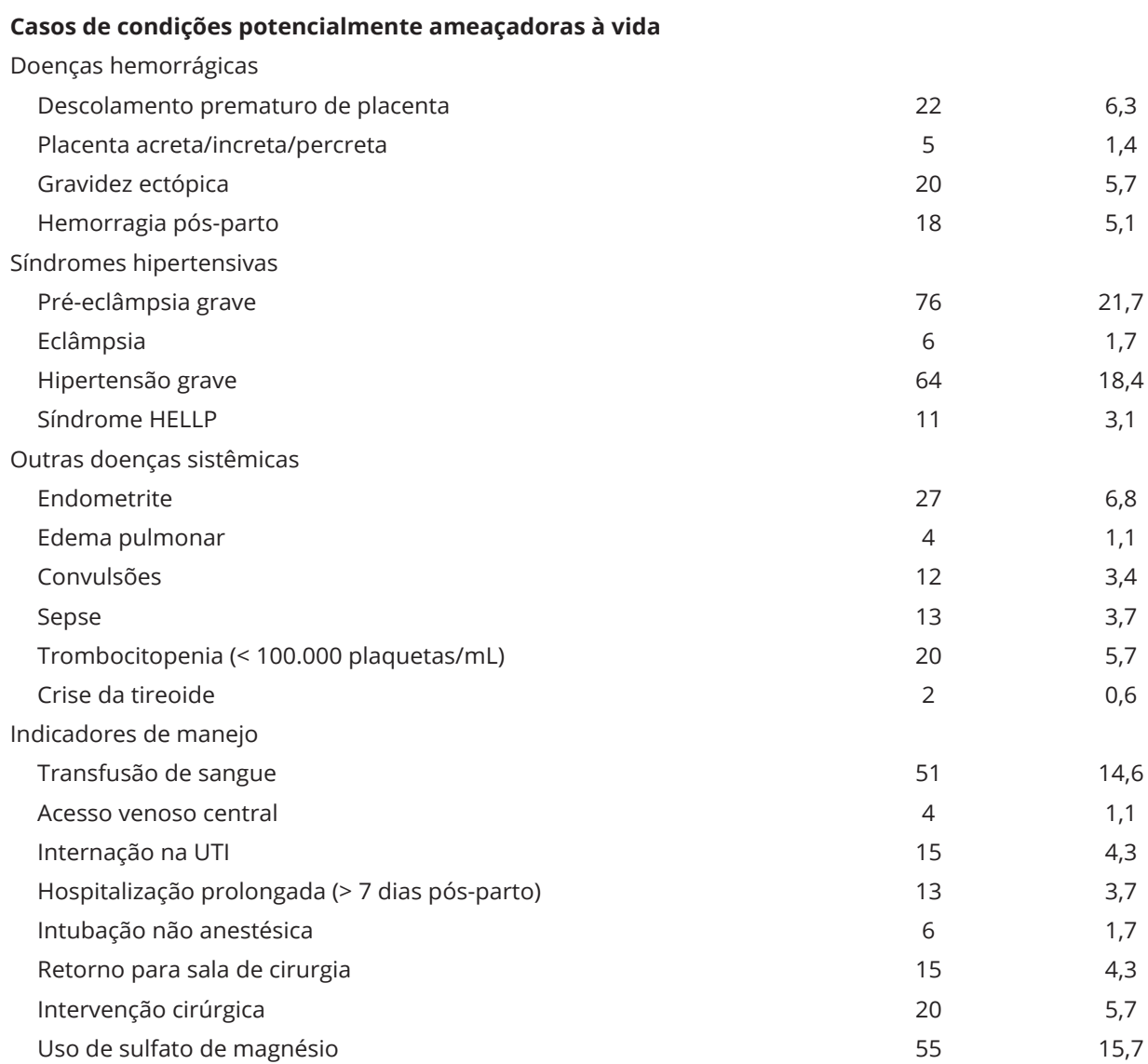

\section{Near miss materno}

Critérios clínicos

Choque

Oligúria não responsiva a líquidos ou diuréticos

Parada cardiorrespiratória

Icterícia na presença de pré-eclâmpsia

Critérios laboratoriais

Saturação de oxigênio ( $<90 \%$ por $\geq 60$ minutos)

Creatinina $\geq 300 \mu \mathrm{mol} / \mathrm{L}$ ou $\geq 3,5 \mathrm{mg} / \mathrm{dL}$

$\mathrm{PH}<7,1$ (acidose grave)

Trombocitopenia grave $(<50.000$ plaquetas $/ \mathrm{mL})$

Coma metabólico

Critérios de manejo

Uso contínuo de drogas vasoativas contínuas

Histerectomia puerperal por infecção ou hemorragia

Transfusão de $\geq 5$ unidades de concentrado de hemácias

Intubação e ventilação por tempo $\geq 60$ minutos não relacionados à anestesia

HELLP: Hemolysis Elevated Liver Enzymes Low Platelets; UTI: unidade de terapia intensiva. 
Tabela 3

Condições sociodemográficas de mulheres com morbidade materna grave (condições potencialmente ameaçadoras à vida/near miss materno) atendidas nos hospitais públicos do Município de Ribeirão Preto, São Paulo, Brasil, 2015/2016.

\begin{tabular}{|c|c|c|}
\hline \multirow[t]{2}{*}{ Variáveis sociodemográficas } & \multicolumn{2}{|c|}{ Morbidade materna grave } \\
\hline & $\mathbf{n}$ & $\%$ \\
\hline \multicolumn{3}{|l|}{ Idade (anos) } \\
\hline$<35$ & 199 & 79,6 \\
\hline$\geq 35$ & 51 & 20,4 \\
\hline \multicolumn{3}{|l|}{ Escolaridade (anos) } \\
\hline$<8$ & 51 & 20,6 \\
\hline$\geq 8$ & 197 & 79,4 \\
\hline \multicolumn{3}{|l|}{ Situação marital } \\
\hline Com companheiro & 202 & 81,1 \\
\hline Sem companheiro & 47 & 19,9 \\
\hline \multicolumn{3}{|l|}{ Raça/Cor } \\
\hline Preta/Parda & 162 & 64,5 \\
\hline Outras * & 89 & 35,5 \\
\hline \multicolumn{3}{|l|}{ Trabalho remunerado } \\
\hline Sim & 123 & 50,2 \\
\hline Não & 122 & 49,8 \\
\hline \multicolumn{3}{|l|}{ Chefe de família } \\
\hline A própria mulher & 54 & 22,1 \\
\hline Companheiro & 138 & 56,6 \\
\hline Outra pessoa & 52 & 21,3 \\
\hline \multicolumn{3}{|l|}{ Classe social ** } \\
\hline$A$ ou $B$ & 29 & 13,2 \\
\hline C & 131 & 59,8 \\
\hline D ou E & 59 & 27,0 \\
\hline
\end{tabular}

* Branca, indígena e oriental/amarela.

** Renda mensal média das classes A (> 20 salários mínimos), B (> 10 e $\leq 20$ salários mínimos), C (> 4 e $\leq 10$ salários mínimos ), D (> 2 e $\leq 4$ salários mínimos) e E ( $\leq 2$ salários mínimos). O salário mínimo na época da pesquisa era de BRL 880,00 (USD 267,81).

\section{Tabela 4}

Antecedentes obstétricos de mulheres com morbidade materna grave (condições potencialmente ameaçadoras à vida/ near miss materno) atendidas nos hospitais públicos do Município de Ribeirão Preto, São Paulo, Brasil, 2015/2016.

\begin{tabular}{lcc}
\hline Antecedentes obstétricos & Morbidade materna grave & \\
& $\mathbf{n}$ & $\%$ \\
\hline Gestações anteriores & 71 & 28,2 \\
0 & 120 & 47,6 \\
$1-2$ & 61 & 24,2 \\
$\geq 3$ & & 37,7 \\
Partos anteriores & 95 & 44,4 \\
0 & 112 & 17,9 \\
$1-2$ & 45 & \\
\hline 3
\end{tabular}

(continua) 
Tabela 4 (continuação)

\section{Antecedentes obstétricos}

Morbidade materna grave

$\%$

Cesarianas prévias

Sim

78

30,9

Não

103

40,9

Partos vaginais prévios

Sim

Não

105

58,0

76

Abortos prévios

Sim

50

27,6

Não

131

72,4

Natimortos anteriores

Sim

Não

175

96,7

Intervalo interpartal (anos)

$<2$

15

10,3

$\geq 2$

130

89,7

Complicações em gestações anteriores

Sim

64

37,6

Não

106

62,4

\section{Tabela 5}

Assistência pré-natal e ao parto de mulheres com morbidade materna grave (condições potencialmente ameaçadoras à vida/near miss materno) atendidas nos hospitais públicos do Município de Ribeirão Preto, São Paulo, Brasil, $2015 / 2016$.

\section{Características do pré-natal e parto}

Morbidade materna grave

n

139

229

30

51

147

171

27

105

50

155

48

14
42,6

57,4

88,4

11,6

25,8

74,2

86,4

13,6

67,7

32,3

71,4

22,1

6,5

(continua) 
Tabela 5 (continuação)

\begin{tabular}{|c|c|c|}
\hline \multirow[t]{2}{*}{ Características do pré-natal e parto } & \multicolumn{2}{|c|}{ Morbidade materna grave } \\
\hline & $\mathbf{n}$ & $\%$ \\
\hline \multicolumn{3}{|l|}{ Gestante de risco } \\
\hline Sim & 143 & 65,6 \\
\hline Não & 75 & 34,4 \\
\hline \multicolumn{3}{|l|}{ Hipertensão } \\
\hline Sim & 94 & 65,7 \\
\hline Não & 49 & 34,3 \\
\hline \multicolumn{3}{|l|}{ Diabetes } \\
\hline Sim & 18 & 12,6 \\
\hline Não & 125 & 87,4 \\
\hline \multicolumn{3}{|l|}{ Sangramento na gestação } \\
\hline Sim & 3 & 2,0 \\
\hline Não & 140 & 98,0 \\
\hline \multicolumn{3}{|l|}{ Infecção urinária } \\
\hline Sim & 7 & 4,9 \\
\hline Não & 136 & 95,1 \\
\hline \multicolumn{3}{|l|}{ Tipo de gestação } \\
\hline Única & 214 & 94,7 \\
\hline Múltipla & 12 & 5,3 \\
\hline \multicolumn{3}{|l|}{ Tipo de parto atual } \\
\hline Vaginal & 91 & 40,4 \\
\hline Cesariana & 134 & 59,6 \\
\hline \multicolumn{3}{|l|}{ Trabalho de parto } \\
\hline Espontâneo & 78 & 30,6 \\
\hline Induzido & 60 & 23,5 \\
\hline Ausente & 83 & 32,6 \\
\hline Aborto/gestação ectópica & 34 & 13,3 \\
\hline
\end{tabular}

UBS: unidades básicas de saúde.

* Privado/Suplementar.

\section{Discussão}

A razão de near miss materno do presente estudo (5,4 casos por mil nascidos vivos) se aproxima dos indicadores observados em pesquisas realizadas no Brasil e em demais países de baixa e média rendas $15,16,17,18,19,20,21$.

Esses resultados mostram que a razão do near miss materno é semelhante ao se comparar distintas regiões, independentemente do desenvolvimento social e econômico local, importando de fato o diagnóstico adequado e a qualidade da assistência ofertada para os casos de complicações maternas graves 8 .

A padronização dos critérios de identificação dos casos de near miss materno, no ano de 2009, viabilizou a comparação da saúde materna entre diferentes regiões, pois antes eram utilizados diferentes critérios elaborados por distintos pesquisadores.

Algumas propostas de classificação consideravam a internação da mulher em unidade de terapia intensiva (UTI) ou a realização de intervenções (histerectomia, transfusão sanguínea etc.) para identificar o caso de near miss materno, outras levavam em conta a ocorrência de determinadas disfunções orgânicas maternas (eclâmpsia, sepse grave, ruptura uterina, dentre outras) 22,23,24.

O grupo técnico da OMS também estruturou indicadores que viabilizam a comparação da saúde materna ao longo do tempo entre diferentes regiões, contribuindo para a identificação dos problemas assistenciais a serem enfrentados 25 . 
Um dos indicadores propostos é a razão do desfecho materno grave, importante para analisar a ocorrência de complicações graves e óbitos na população atendida. A razão do desfecho materno grave viabiliza o entendimento da complexidade da assistência necessária à população assistida e o gerenciamento dos recursos adequados para a oferta do cuidado obstétrico de qualidade 6 .

Nos hospitais analisados, a razão do desfecho materno grave foi de 6,0 casos por mil nascidos vivos, valor semelhante a alguns estudos de base hospitalar realizados no Brasil e em outros países de média e baixa rendas 16,20,21. A razão do desfecho materno grave pode variar de acordo com a realidade pesquisada, mas espera-se que esse valor esteja em torno de 7,5 casos por mil nascidos vivos. Quanto menor o indicador, melhores são as condições de saúde materna e da assistência ofertada no local pesquisado 6 .

A razão entre o near miss materno e a mortalidade materna consiste em um indicador que reflete a qualidade do atendimento obstétrico, sendo que elevados valores apontam para uma assistência adequada e prevenção de óbitos entre as mulheres com near miss materno ${ }^{6}$.

Nas maternidades pesquisadas em Ribeirão Preto, ocorreram 9,5 casos de near miss materno para cada óbito, valor superior ao encontrado pelo estudo da Rede Nacional de Vigilância de Morbidade Materna Grave 8 e aproximadamente o dobro dos valores encontrados em pesquisas realizadas em estados do Nordeste 16,26, o que mostra a disparidade da assistência à saúde materna ao serem comparadas as diferentes regiões brasileiras.

Um estudo de tendência temporal realizado nas diferentes regiões brasileiras, mostrou que a assistência obstétrica inadequada está diretamente relacionada a uma tendência de aumento das taxas de near miss materno observada nas regiões Norte e Nordeste. Em contraponto, houve redução da razão do near miss materno nas regiões Sudeste e Centro-oeste, decorrente da melhoria efetiva da assistência à saúde materna nestes locais 27.

A RMM encontrada no presente trabalho $(57,1)$ corrobora o indicador nacional de 58,9 óbitos por 100 mil nascidos vivos (Departamento de Informática do SUS. Indicadores de mortalidade e indicadores de nascidos vivos. http://www2.datasus.gov.br/DATASUS/index.php?area=0205, acessado em 10/Mar/2019), mesmo sendo realizado em uma região mais rica e desenvolvida do país (Instituto Brasileiro de Geografia e Estatística. População e economia. https://cidades.ibge.gov.br/brasil/sp/ribei rao-preto/panorama, acessado em 10/Mar/2019), sugerindo que a qualidade do cuidado prestado e, não apenas o acesso ao serviço, seja o principal aspecto da assistência ao parto a ser discutido no país.

O Brasil está na fase III da transição obstétrica (RMM entre 299 e 50 óbitos por 100 mil nascidos vivos), e as mortes maternas que ocorrem nos países desta fase estão relacionadas principalmente à qualidade da assistência às mulheres no período gravídico-puerperal 18, o que mostra a relevância em se melhorar a assistência obstétrica nos serviços pesquisados.

Os dois óbitos maternos que ocorreram durante este estudo foram por causa hemorrágica. As mortes relacionadas com hemorragia pós-parto são características de países de baixa renda e são altamente evitáveis desde que o manejo adequado seja realizado. Esses casos sugerem fortemente importantes dificuldades assistenciais nos serviços de saúde 8,18, embora a taxa de mortalidade entre casos de desfecho materno grave tenha sido de 9,5\% e, portanto, inferior à encontrada em um estudo multicêntrico brasileiro $(15,4 \%) 8$, o que mostra uma melhor assistência às urgências obstétricas no município pesquisado.

A realização de auditoria, baseando-se nos casos de near miss materno é fundamental, pois permite o levantamento de problemas referentes à assistência recebida pelas mulheres com complicações graves. Esse conhecimento pode viabilizar a melhoria do cuidado ofertado, prevenindo assim o agravamento da morbidade e consequente óbito na gestação, parto e pós-parto. Nesse contexto, a auditoria da qualidade do cuidado apenas com base nos casos de óbitos seria ineficaz em decorrência do reduzido número absoluto de casos em regiões mais desenvolvidas 21,28 .

Neste trabalho, as complicações hipertensivas e hemorrágicas apresentaram destaque entre os casos de morbidade materna grave, e estas são as principais causas de morbidade e morte materna no território brasileiro 8,29,30.

Observa-se no presente estudo uma maior proporção de mulheres pretas e pardas em relação às mulheres com outras características de cor da pele, o que pode contribuir para o maior número de complicações hipertensivas. 
Um estudo realizado na Califórnia, Estados Unidos, mostrou associação entre mulheres de etnia afro-americana e a ocorrência de pré-eclâmpsia, o que pode ser explicado por fatores genéticos e biológicos. Entretanto, os autores destacam a necessidade de estudos mais aprofundados para uma melhor elucidação sobre tal relação 31. Pesquisas realizadas na Holanda e no Reino Unido também encontraram associação entre a incidência de eclâmpsia e etnia, principalmente no grupo de mulheres africanas 32,33 .

Como exemplo da importância da análise dos casos, pôde-se observar nos hospitais de Ribeirão Preto a alta prevalência de pré-eclâmpsia, mas a ocorrência de eclâmpsia foi extremamente baixa, o que indica uma adequada assistência para estes casos. Medidas desenvolvidas em tempo hábil, tais como a administração de sulfato de magnésio e a retirada da placenta, por exemplo, são essenciais para a prevenção de convulsões e piora do prognóstico ${ }^{34}$.

A predominância dos distúrbios hipertensivos entre os casos de complicações maternas graves também mostra a necessidade de melhorias no rastreio da patologia durante o pré-natal 20.

Nesta análise, observou-se que 11,6\% das mulheres entrevistadas não realizaram nenhuma consulta pré-natal e dentre aquelas que fizeram o acompanhamento, 25,8\% realizaram menos de seis consultas durante a gestação. Estudos apontam que a qualidade da assistência pré-natal, a baixa cobertura e a não realização de ao menos seis consultas são conhecidos fatores de risco para o near miss materno 35,36 .

Outro importante fator associado ao desenvolvimento de graves complicações maternas é a realização de cesárea 26,35,37,38. O Brasil é conhecido por ser um país com elevada incidência de cesarianas e com tendência a crescimento da frequência do procedimento 30,39 .

Um estudo multipaíses mostrou associação entre a cesárea e a maior incidência de óbitos maternos, hemorragia, histerectomia e morbidade materna grave 38 .

Entretanto, é importante destacar também que a complicação materna, em muitas situações, demanda a realização do parto cirúrgico. Como uma das instituições pesquisadas é referência para a gestação de alto risco, a maior frequência de cesarianas pode ser decorrente não apenas de indicações indevidas, mas também de demandas reais devido a graves complicações obstétricas atendidas na instituição.

A existência de mais de um critério de elegibilidade para uma mesma mulher, verificado entre as mulheres hospitalizadas em Ribeirão Preto, mostra a complexidade da gestão da assistência necessária para estas situações, além do aumento da probabilidade de óbito na medida em que cresce o número de marcadores de gravidade para um mesmo caso 16,40 .

Uma vantagem deste trabalho é o fato de viabilizar o conhecimento das morbidades maternas graves em uma região de alta renda, condição que difere da maior parte do território brasileiro, mostrando que os indicadores de morbidade não mudam, mesmo comparando realidades socioeconômicas distintas. A qualidade da assistência é o ponto central para a redução das complicações maternas graves.

Uma limitação diz respeito ao fato de as mulheres com menos de 18 anos não terem entrado no estudo de vigilância, pois o PRENACEL pesquisou apenas mulheres com 18 anos ou mais. O que significa que foram excluídas mulheres abaixo dos 18 anos, sabidamente uma faixa etária que pode apresentar maior risco obstétrico.

Além disso, não foi possível analisar os fatores associados às morbidades maternas graves. $\mathrm{O}$ estudo de vigilância envolveu todas as mulheres com condições potencialmente ameaçadoras à vida e near miss materno atendidas nos hospitais públicos do município, o que incluiu mulheres de todo o município e de cidades vizinhas, já que Ribeirão Preto é referência na prestação de serviços de média e alta complexidades para toda a região.

Com isso, a heterogeneidade das mulheres pesquisadas em relação aos locais de origem e consequentes contextos sociais, econômicos e culturais inviabilizou a obtenção de um grupo de comparação, pois o estudo principal (PRENACEL) pesquisou apenas mulheres oriundas de 20 UBS do município, o que limitou a utilização destas mulheres sem complicações maternas como grupo de comparação.

Estudos de vigilância das morbidades maternas graves usando os critérios estabelecidos pela OMS podem ser realizados em diferentes serviços de saúde para a avaliação do cuidado realizado. $O$ entendimento sobre a frequência dos casos de complicações maternas graves e os diagnósticos mais comuns 
é relevante para compreender quais são os entraves na assistência, desde a atenção básica até a alta complexidade, que não estão conseguindo intervir para que as morbidades maternas não compliquem e possam levar ao risco de vida e possível óbito.

Esse entendimento pode contribuir para a elaboração de protocolos assistenciais que determinem diretrizes para a assistência às complicações maternas graves, permitindo verificar o ponto de falha antes do óbito, o que pode contribuir para a recomendação de intervenções que salvem vidas 16.

\section{Conclusões}

Os resultados deste trabalho mostram uma importante frequência de casos de condições potencialmente ameaçadoras à vida e near miss materno em Ribeirão Preto, e a RMM encontrada é semelhante ao indicador nacional. O adoecimento materno decorrente das complicações hipertensivas destaca-se entre as causas de morbidade grave, e a hemorragia foi a principal causa de mortalidade. Os resultados apresentados evidenciam o fato de que a qualidade da assistência obstétrica no Brasil é determinante para o grave adoecimento materno, inclusive nas localidades de maior renda.

$\mathrm{O}$ fato de muitas mulheres com morbidade materna grave terem apresentado mais de um critério de elegibilidade para condições potencialmente ameaçadoras à vida ou near miss materno mostra a complexidade dos casos, o que demanda cuidado qualificado e em tempo hábil para a proteção da saúde materna.

\section{Colaboradores}

Todos os autores contribuíram na concepção, projeto, análise e interpretação dos dados; na redação do artigo e revisão crítica relevante do conteúdo intelectual; e na aprovação final da versão a ser publicada. Também são responsáveis por todos os aspectos do trabalho na garantia da exatidão e integridade de qualquer parte da obra.

\section{Informações adicionais}

ORCID: Magna Santos Andrade (0000-00020557-2169); Livia Pimenta Bonifacio (0000-00024309-0304); Jazmin Andrea Cifuentes Sanchez (0000-0002-4552-0443); Livia Oliveira-Ciabati (0000-0002-7163-9456); Fabiani Spessoto Zaratini (0000-0002-7758-4388); Ana Carolina Arruda Franzon (0000-0003-4333-3326); Vicky Nogueira Pileggi (0000-0003-2284-010X); Giordana Campos Braga (0000-0002-0719-056X); Mariana Fernandes (0000-0002-4551-7017); Carolina Sales Vieira (0000-0003-2933-5755); Joao Paulo Souza (00000002-2288-4244); Elisabeth Meloni Vieira (00000002-5229-3904).

\section{Agradecimentos}

O PRENACEL foi financiado pelo Programa Ciência sem Fronteiras (CSF-PAJT 2514/2013) da Coordenação de Aperfeiçoamento de Pessoal de Nível Superior/Conselho Nacional de Desenvolvimento Científico e Tecnológico (no processo 23038.007622/2013-60) e Fundação de Amparo à Pesquisa do Estado de São Paulo (processo 2015/16932-5), com apoio institucional da Organização Mundial da Saúde. 


\section{Referências}

1. Alkeman L, Chou D, Hogan D, Zhang S, Moler A, Gemmill A, et al. Global, regional, and national levels and trends in maternal mortality between 1990 and 2015, with scenario-based projections to 2030: a systematic analysis by the UN Maternal Mortality Estimation InterAgency Group. Lancet 2016; 387:462-74.

2. Lozano R, Wang H, Foreman KJ, Rajaratnam JK, Naghavi M, Marcus JR, et al. Progress towards Millennium Development Goals 4 and 5 on maternal and child mortality: an updated systematic analysis. Lancet 2011; 378:1139-65.

3. United Nations. Millennium Development Goals and beyond 2015. New York: United Nations; 2013.

4. World Health Organization. World health statistics 2017: monitoring health fot the SDGs, susteinable development goals. Geneva: World Health Organization; 2017.

5. Say L, Souza JP, Pattinson RC. Maternal near miss - towards a standard tool for monitoring quality of maternal health care. Best Pract Res Clin Obstet Gynaecol 2009; 23:287-96.

6. World Health Organization. Evaluating the quality of care for severe pregnancy complications - The WHO near-miss approach for maternal health. Geneva: World Health Organization; 2011.

7. Kalisa R, Rulisa S, van den Akker T, van Roosmalen J. Maternal near miss and quality of care in a rural Rwandan hospital. BMC Pregnancy Childbirth 2016;16:324.

8. Cecatti JG, Costa ML, Haddad SM, Parpinelli MA, Souza JP, Sousa MH, et al. Network for surveillance of severe maternal morbidity: a powerful national collaboration generating data on maternal health outcomes and care. BJOG 2016;123:946-53.

9. Souza JP, Gülmezoglu AM, Vogel J, Carroli G, Lumbiganon P, Qureshi Z, et al. Moving beyond essential interventions for reduction of maternal mortality (the WHO Multicountry Survey on Maternal and Newborn Health): a cross-sectional study. Lancet 2013; 381:174755.

10. Nakimuli A, Nakubulwa S, Kakaire O, Osinde MO, Mbalinda SN, Nabirye RC, et al. Maternal near misses from two referral hospitals in Uganda: a prospective cohort study on incidence, determinants and prognostic factors. BMC Pregnancy Childbirth 2016; 16:24.

11. Andrade MS, Vieira EM. Itinerários terapêuticos de mulheres com morbidade materna grave. Cad Saúde Pública 2018; 34:e00091917.

12. Firoz T, Chou D, Dadelszen P, Agrawal P, Vanderkruik R, Tunçalp O, et al. Measuring maternal health: focus on maternal morbidity. Bull World Health Organ 2013; 91:794-96.

13. Oliveira-Ciabati L, Vieira CS, Franzon ACA, Alves D, Zaratini FS, Braga GC, et al. PRENACEL - a mHealth messaging system to complement antenatal care: a cluster randomized trial. Reprod Health 2017; 14:146.
14. Departamento de Atenção Básica, Secretaria de Atenção à Saúde, Ministério da Saúde. Atenção ao pré-natal de baixo risco. Brasília: Ministério da Saúde; 2012. (Série A. Normas e Manuais Técnicos) (Cadernos de Atenção Básica, 32).

15. Santana DS, Cecatti JG, Surita FG, Silveira C, Costa ML, Souza JP, et al. Twin pregnancy and severe maternal outcomes. Obstet Gynecol 2016; 127:631-41.

16. Galvão LPL, Alvim-Pereira F, Mendonça CMM, Menezes FEF, Góis KAN, Ribeiro Jr. $\mathrm{RF}$, et al. The prevalence of severe maternal morbidity and near miss and associated factors in Sergipe, Northeast Brazil. BMC Pregnancy Childbirth 2014; 14:25.

17. Zanette E, Parpinelli MA, Surita FG, Costa ML, Haddad SM, Sousa MH, et al. Maternal near miss and death among women with severe hypertensive disorders: a Brazilian multicenter surveillance study. Reprod Health 2014; 11:4.

18. Chaves SC, Cecatti JG, Carroli G, Lumbiganon P, Hogue CJ, Mori R, et al. Obstetric transition in the World Health Organization Multicountry Survey on Maternal and Newborn Health: exploring pathways for maternal mortality reduction. Rev Panam Salud Pública 2015; 37:203-10.

19. Lotufo FA, Parpinelli M, Haddad SM, Surita FG, Cecatti JG. Applying the new concept of maternal near-miss in an intensive care unit. Clinics 2012; 67:225-20.

20. Ghazivakili Z, Lotfi R, Kabir K, Norouzi Nia R, Rajabi Naeeni M. Maternal near miss approach to evaluate quality of care in Alborz province, Iran. Midwifery 2016; 41:118-24.

21. Jabir M, Abdul-Salam I, Suheil DM, Al-Hilli W, Abul-Hassan S, Al-Zuheiri A, et al. Maternal near miss and quality of maternal health care in Baghdad, Iraq. BMC Pregnancy Childbirth 2013; 13:11.

22. Bouvier-Colle MH, Salanave B, Ancel PY, Varnoux N, Fernandez H, Papaverina E, et al. Obstetric patients in intensive care units and maternal mortality. Regional Teams for the Survey. Eur J Obstet Gynecol Reprod Biol 1996; 65:121-5.

23. Pattinson RC, Hall M. Near misses: a useful adjunct to maternal death enquiries. Br Med Bull 2003; 67:231-43.

24. Waterstone M, Bewley S, Wolfe C. Incidence and predictors of severe obstetric morbidity: case-control study. BMJ 2001; 322:1089-94.

25. Pattinson R, Say L, Souza JP, Broek N, Rooney C. WHO maternal death and near-miss classifications. Bull World Health Organ 2009; 87:734.

26. Madeiro AP, Rufino AC, Lacerda EZG, Brasil LG. Incidence and determinants of severe maternal morbidity: a transversal study in a referral hospital in Teresina, Piaui, Brazil. BMC Pregnancy Childbirth 2015; 15:210. 
27. Carvalho BAS, Andrade AGBF, Dantas AS, Figueiredo IM, Silva JA, Rosendo TS, et al. Tendência temporal do Near Miss materno no Brasil entre 2000 e 2012. Rev Bras Saúde Mater Infant (Online) 2019; 19:115-24.

28. Cecatti JG, Souza JP, Parpinelli MA, de Sousa $\mathrm{MH}$, Amaral E. Research on severe maternal morbidities and near-misses in Brazil: what we have learned. Reprod Health Matters 2007; 15:125-33.

29. Silva JMP, Fonseca SC, Dias MAB, Izzo AS, Teixeira GP, Belfort PP. Conceitos, prevalência e características da morbidade materna grave, near miss, no Brasil: revisão sistemática. Rev Bras Saúde Mater Infant (Online) 2018; 18:735.

30. Departamento de Análise de Situação em Saúde, Secretaria de Vigilância em Saúde, Ministério da Saúde. Saúde Brasil 2013: uma análise da situação de saúde e das doenças transmissíveis relacionadas à pobreza. Brasília: Ministério da Saúde; 2014.

31. Caughey AB, Stotland NE, Washington AE, Escobar GJ. Maternal ethnicity, paternal ethnicity, and parental ethnic discordance: predictors of pre-eclampsia. Obstet Gynecol 2005; 106:156-61.

32. Zwart JJ, Jonkers MD, Richters A, Ory F, Bloemenkamp KW, Duvekot JJ, et al. Ethnic disparity in severe acute maternal morbidity: a nationwide cohort study in the Netherlands. Eur J Public Health 2010; 21:229-34.

33. Knight M, Kurinczuk JJ, Spark P, Brocklehurst P. Inequalities in maternal health: national cohort study of ethnic variation in severe maternal morbidities. BMJ 2009; 338:b542.

34. Duley L, Gülmezoglu AM, Henderson-Smart DJ, Chou D. Magnesium sulphate and other anticonvulsants for women with pre-eclampsia. Cochrane Database Syst Rev 2010; (11):CD000025.
35. Lima THB, Amorim MM, Samir Kassar SB, Katz L. Maternal near miss determinants at a maternity hospital for high-risk pregnancy in northeastern Brazil: a prospective study. BMC Pregnancy Childbirth 2019; 19:271.

36. Domingues RMSM, Dias MAB, Schilithz AOC, Leal MC. Factors associated with maternal near miss in childbirth and the postpartum period: findings from the birth in Brazil National Survey, 2011-2012. Reprod Health 2016; 13 Suppl 3:187-97.

37. Norhayati MN, Nik Hazlina NH, Sulaiman Z, Azman NY. Severe maternal morbidity and near misses in tertiary hospitals, Kelantan, Malaysia: a cross-sectional study. BMC Public Health 2016; 16:229.

38. Souza JP, Cecatti JG, Faundes A, Morais SS, Villar J, Carroli G, et al. Maternal near miss and maternal death in the World Health Organization's 2005 global survey on maternal and perinatal health. Bull World Health Organ 2010; 88:113-9.

39. Barros FC, Matijasevich A, Maranhão AGK, Escalante Juan JJ, Rabello Neto DL, Fernandes RM, et al. Cesarean sections in Brazil: will they ever stop increasing? Rev Panam Salud Pública 2015; 38:217-25.

40. Souza JP, Cecatti JG, Haddad SM, Parpinelli MA, Costa ML, Leila Katz, et al. The WHO maternal near-miss approach and the maternal severity index model (msi): tools for assessing the management of severe maternal morbidity. PLoS One 2012; 7:e44129. 
Abstract

This study analyzed the occurrence of severe maternal morbidity, the most frequent diagnostic criteria, and the quality of obstetric care in public hospitals in Ribeirão Preto, São Paulo State, Brazil. A quantitative surveillance survey of severe maternal morbidity used World Health Organization (WHO) criteria for potentially life-threatening conditions and maternal near miss. Cases were identified from August 1, 2015, to February 2, 2016. The sample included 259 women with severe maternal morbidity (potentially life-threatening conditions/maternal near miss) during the gestational and postpartum cycle, hospitalized for childbirth in the four public institutions providing obstetric care in the city. The descriptive analysis was based on absolute and relative rates of diagnostic criteria for potentially life-threatening conditions and maternal near miss, besides description of the women in the sample (sociodemographic characteristics, obstetric history, and prenatal and childbirth care). Quality of care indicators set by the WHO based on morbimortality were also calculated. There were 3,497 deliveries, 3,502 live births in all the hospitals in the city, two maternal deaths, and 19 maternal near miss. Maternal near miss ratio was 5.4 cases per 1,000 live births, and the maternal mortality ratio was 57.1 deaths per 100,000 live birth. The mortality rate among cases with severe maternal outcome (maternal near miss plus maternal death) was $9.5 \%$. The study revealed important potentially life-threatening conditions and maternal near miss rates. The occurrence of deaths from hemorrhagic causes highlights the need to improve the quality of obstetric care. The findings can potentially help improve local policy for obstetric care.

Healthcare Near Miss; Maternal Death; Morbidity

\section{Resumen}

El objetivo de este estudio es investigar la ocurrencia de morbilidad materna grave, así como los criterios para los diagnósticos más frecuentes y la calidad de la asistencia obstétrica en hospitales públicos de Ribeirão Preto, São Paulo, Brasil. Se trata de una investigación cuantitativa de vigilancia de la morbilidad materna grave, utilizando criterios de la Organización Mundial de la Salud (OMS) sobre condiciones potencialmente amenazadoras para vida $y$ near miss materno. Los casos se identificaron entre el 1 de agosto de 2015 y 2 de febrero de 2016. Participaron 259 mujeres con morbilidad materna grave (condiciones potencialmente amenazadoras para vida/near miss materno) durante el ciclo embarazo, parto y puerperio, internadas para el parto en cuatro instituciones públicas con asistencia obstétrica en el municipio. Se realizó un análisis descriptivo, a partir de frecuencias absolutas y relativas con criterios diagnósticos para condiciones potencialmente amenazadoras para vida $y$ near miss materno, además de la descripción de las mujeres investigadas (caracterizaciones sociodemográficas, antecedentes obstétricos, asistencia prenatal y al parto). También se calcularon indicadores de calidad de asistencia establecidos por la OMS a partir de la morbimortalidad. Se produjeron 3.497 partos, 3.502 nacidos vivos en todos los hospitales del municipio, 2 muertes maternas y 19 near miss materno. La razón del near miss materno fue de 5,4 casos por 1.000 nacidos vivos y la razón de mortalidad materna fue 57,1 óbitos por 100.000 nacidos vivos. La tasa de mortalidad entre los casos con desenlace materno grave (sumados a los casos de near miss materno y muerte materna) fue de un 9,5\%. Se observó una importante frecuencia de casos de condiciones potencialmente amenazadoras para vida $y$ near miss materno en el presente estudio. La ocurrencia de óbitos por causas hemorrágicas expone la necesidad de una mejora de la calidad en la asistencia prestada. Los resultados podrán contribuir a la mejora de la política local de asistencia obstétrica.

Near Miss Salud; Muerte Materna; Morbilidad
Recebido em 22/Mai/2019

Versão final reapresentada em 07/Nov/2019

Aprovado em 12/Dez/2019 